\title{
Adaptive Behavior Ratings Correlate with Symptomatology and IQ Among Individuals with High-Functioning Autism Spectrum Disorders
}

\author{
Lauren Kenworthy ${ }^{1,2}$, Laura Case ${ }^{2}$, Madeline B. Harms ${ }^{2}$, Alex Martin ${ }^{2}$, and Gregory L. \\ Wallace ${ }^{2}$ \\ ${ }^{1}$ Center for Autism Spectrum Disorders, Children's National Medical Center, Washington, DC \\ ${ }^{2}$ Laboratory of Brain \& Cognition, National Institute of Mental Health, Bethesda, MD
}

\begin{abstract}
Caregiver report on the Adaptive Behavior Assessment System-II (ABAS) for 40 high-functioning individuals with autism spectrum disorders (ASD) and 30 typically developing (TD) individuals matched for age, IQ, and sex ratio revealed global adaptive behavior deficits in ASD, with social skills impairments particularly prominent. Within the ASD group, adaptive communication skills were positively related to IQ while global adaptive functioning was negatively associated with autism symptomatology. Autistic behavior ratings related negatively to ABAS scores in the TD but not the ASD group. This investigation demonstrates: the utility of an adaptive functioning checklist for capturing impairments, even in high-functioning individuals with ASD; and that a relationship between social abilities and autism exists independently of intelligence.
\end{abstract}

\section{Keywords}

autism; adaptive behavior; social skills; IQ; symptomatology; Asperger's syndrome

\begin{abstract}
Adaptive functioning encompasses those behaviors critical to living independently, including daily living skills (e.g., dressing and grooming oneself), social skills, and communication skills. Children with Autism Spectrum Disorders (ASD) display adaptive behavior impairments across all domains. In addition to deficits in communication and socialization, children with ASD display poorer daily living skills than their unaffected peers and than children with other developmental disorders (Klin, Saulnier, Sparrow, Cicchetti, \& Volkmar, 2007; Gillham, Carter, Volkmar, \& Sparrow, 2000; Perry, Flanagan, Dunn Geier, \& Freeman, 2009). Standardized measures of adaptive behavior can be useful in diagnosing ASD because, unlike the most commonly utilized diagnostic measures, they provide agereferenced information about a child's communication, socialization, and other behaviors (Gillham et al., 2000). Indeed, when clinicians obtain discrepant diagnoses from the Autism Diagnostic Interview (ADI) and Autism Diagnostic Observation Schedule (ADOS),
\end{abstract}

Correspondence concerning this article should be addressed to: Lauren Kenworthy, Center for Autism Spectrum Disorders, Children's National Medical Center, 15245 Shady Grove Road, South Building, Suite 350, Rockville, MD 20850, Phone: 301-765-5430, Fax: 301-765-5497, 1kenwort@cnmc.org.

Lauren Kenworthy, Center for Autism Spectrum Disorders, Children's National Medical Center, Washington, DC, USA

Laura Case, Laboratory of Brain \& Cognition, National Institute of Mental Health, Bethesda, MD, USA

Madeline B. Harms, Laboratory of Brain \& Cognition, National Institute of Mental Health, Bethesda, MD, USA

Alex Martin, Laboratory of Brain \& Cognition, National Institute of Mental Health, Bethesda, MD, USA

Gregory L. Wallace, Laboratory of Brain \& Cognition, National Institute of Mental Health, Bethesda, MD, USA 
assessment of adaptive functioning significantly improves diagnostic classification accuracy (Tomanik, Pearson, Loveland, Lane, \& Shaw, 2007).

It is no surprise that adaptive skill assessments are informative in making diagnostic decisions, as adaptive socialization skills in particular have obvious relevance to the social interaction deficits that lie at the core of ASD. Previous investigations of the adaptive behavior profile in ASD consistently reveal impairment in socialization skills while reporting somewhat more variable findings regarding other adaptive domains. In several studies the socialization domain of adaptive behavior shows more impairment than other adaptive domains (Bolte \& Poustka, 2002; Carter et al., 1998; Perry et al., 2009). Using age equivalent scores, Carter et al. (1998) show a relative weakness in Vineland Adaptive Behavior Scales (VABS) Socialization and a relative strength in Daily Living Skills in verbal and nonverbal children with autism. Liss et al. (2001) find that relative to controls with developmental language disorders, children with autism are significantly more impaired in Socialization and Daily Living Skills, but not Communication. The effect is more pronounced in those with high-functioning ASD, and reflects the fact that the language-disordered group is impaired on the Vineland Communication domain (the highfunctioning ASD adaptive profile is relatively flat with standard scores in the 70s (i.e., nearly two standard deviations below the mean) across the Communication, Daily Living Skills and Socialization domains). Fenton and colleagues (2003) find a relatively homogeneous adaptive behavior profile in children with autism and children with severe developmental delay.

Beyond informing diagnosis, Klin et al. (2007) find that adaptive abilities capture an aspect of functioning that is distinct from autism symptoms, or disabilities, and provide important behavioral markers and targets for intervention that are not captured by IQ and have been under-investigated in high-functioning children and adolescents on the autism spectrum. Adaptive functioning is a strong predictor of future language acquisition in 2-3 year olds with ASD (Thurm, Lord, Lee, \& Newschaffer, 2007), but is generally independent of intelligence in children with high-functioning ASD. While IQ can be predictive of adaptive behavior in low-functioning autism (e.g., Liss et al., 2001; Bolte \& Poustka, 2002), it is not typically predictive of adaptive behavior in individuals with high-functioning ASD, particularly regarding socialization skills (Bolte \& Poustka, 2002; Freeman, Del'Homme, Guthrie, \& Zhang, 1999; Liss et al., 2001; Volkmar et al., 1987; Kenworthy et al., 2005). The initial IQ of children with ASD predicts their rate of score increase in the skill areas of Communication and Daily Living Skills, but not Socialization skills, suggesting a particular dissociation between IQ and social ability in autism (Freeman et al., 1999), as well as the close relationship of IQ, particularly VIQ, to many language based adaptive skills captured by the Communication domain (Black, Wallace, Sokoloff, \& Kenworthy, 2009). In another study, age and IQ positively correlate with adaptive skills in autism, but increases in IQ are associated with smaller increases in social functioning in children with autism than in children with intellectual disability (Schatz \& Hamdan-Allen, 1995). Furthermore, Klin et al. (2007) find negative relationships between age and standard adaptive behavior scores, suggesting that children with high-functioning ASD do not make gains in adaptive behaviors over time that are commensurate with their typically developing peers. Thus, adaptive behavior, particularly socialization skill, is more severely impaired than expected based on IQ in children with high-functioning ASD, and it does not show a positive developmental trajectory over time. Measuring and treating social adaptive skills in high-functioning ASD as children move into adolescence is an important step for improving long-term outcomes.

The purpose of this paper is to explore the general utility of a relatively new instrument, the Adaptive Behavior Assessment System (ABAS-II, Harrison \& Oakland, 2003), for assessing adaptive functioning in individuals with ASD. It is an adaptive behavior checklist which can 
be completed quickly and independently by a parent or caregiver. The ABAS has been applied to autism very infrequently in the extant literature (Bassin \& Gorman, 2003; Pfeiffer, Kinnealey, Reed, \& Herzberg, 2005). The ABAS-II manual does provide data on several groups of preschool age children with Pervasive Developmental Disorder-Not Otherwise Specified and autism and one group of 32 older children (ages 5-18) with autism. Although IQ is not indicated, the groups appear to be largely comprised of low functioning children. This investigation complements that data by investigating whether the ABAS effectively characterizes adaptive behavior in high-functioning ASD.

We compare the ABAS adaptive behavior profile of high-functioning individuals with ASD to typically developing (TD) controls matched on age, IQ and sex ratio, and we examine the relationship of these profiles with intelligence, age, and autism symptomatology. Based on previous findings with the VABS, we hypothesize that the ABAS scores in our highfunctioning ASD group will be depressed relative to matched controls and to their own intelligence. We expect a relative weakness within the high-functioning ASD group on the ABAS measure of socialization. Finally, we predict that adaptive behavior as measured by the ABAS will be related in high-functioning ASD to autism symptoms and behaviors.

\section{Methods}

\section{Participants}

Participants were 30 TD individuals ( 26 males, 4 female) between 12 and 21 years of age and 40 high-functioning individuals with an ASD (36 males, 4 females) between 12 and 22 years of age. All 40 participants with ASD met diagnostic criteria based upon DSM-IV criteria (25 Asperger's syndrome, 8 high-functioning autism, 5 pervasive developmental disorder-not otherwise specified, and 2 with an unspecified ASD because of missing data). Thirty-eight participants received the Autism Diagnostic Interview (ADI or ADI-R; LeCouteur et al., 1989; Lord, Rutter, \& LeCouteur, 1994) and 37 participants received the Autism Diagnostic Observation Schedule (ADOS; Lord et al., 2000) administered by a trained, research-reliable clinician. Participants received either module $3(n=12)$ or $4(n=25)$ of the ADOS. Thirty-six participants received both the ADI and the ADOS. All of these participants' scores met cut-off for the category designated as 'Broad ASD' according to criteria established by the NICHD/NIDCD Collaborative Programs for Excellence in Autism (CPEA; see Lainhart et al., 2006). Exclusion criteria for the ASD group included any known co-morbid medical conditions, such as fragile $\mathrm{X}$ syndrome or other genetic disorders, brain trauma/injury, and any neurological disorder that might affect cognitive functioning. TD participants were recruited from the community and parents of all TD participants underwent telephone screenings. TD participants were excluded from participation if they had ever received mental health treatment for anxiety, depression, or any other psychiatric condition, taken psychiatric medications, required special services in school, been diagnosed with a genetic disorder or neurological disorder, or had brain trauma/injury that could potentially affect cognitive functioning and/or brain development. IQ scores were obtained from all but one of the TD participants. All Full Scale IQ scores (FSIQ) were above 80, as measured by the Wechsler Abbreviated Scale of Intelligence (ASD: $n=28, T D: n=27$ ), Wechsler Adult Intelligence Scale-III (ASD: $n=2$ ), Wechsler Intelligence Scale for ChildrenIII (ASD: $n=2$ ) or Wechsler Intelligence Scale for Children-IV (ASD: $n=8$ ). Participant groups did not differ in terms of FSIQ, age, or sex ratio (see Table 1). Informed assent and consent were obtained from all participants and/or their parent/guardian when appropriate.

\section{Measures}

1. Diagnostic Measures-Diagnoses of ASD participants were confirmed with the ADI or ADI-R (LeCouteur et al., 1989; Lord et al., 1994) and ADOS (Lord et al., 1999). The 
ADI is a detailed parent or caregiver interview of developmental history and autism symptoms. Scores are aggregated into symptom clusters that correspond to DSM-IV criteria for a diagnosis of autism; ADI reciprocal social interaction, verbal communication, and repetitive behaviors were included in analyses. The ADOS is a structured play and conversational interview that includes a series of social presses and other opportunities to elicit symptoms of an ASD. Scores are aggregated into symptom clusters that correspond to sums of observed symptoms. The ADOS symptom clusters of social interaction, communication, and stereotyped behaviors were included in analyses.

2. Adaptive Behavior Assessment System-II (ABAS-II)-The ABAS is a measure of adaptive behavior with national standardization samples representative of the Englishspeaking US population. The ABAS manual (Harrison \& Oakland, 2003) documents good test-retest reliability coefficients (mostly in the .90 s for the measure as a whole) and convergent validity with the VABS in a sample of 44 TD individuals. The parent report school age form of the ABAS-II (Harrison \& Oakland, 2003) used in this study was standardized on 1670 individuals and provided information in the skill areas of Communication, Community Use, Functional Academics, Home Living, Health/Safety, Leisure, Self-direction, Social (skills), and Work (for participants 17 and older). Work skill area scores were omitted from all analyses because the majority of study participants did not have work experience and were under 17 years of age. Skill area scores are presented as norm-referenced scaled scores $(\mathrm{M}=10 ; \mathrm{SD}=3)$, and are aggregated into three composite scores: Conceptual (CON; Communication, Functional Academics, Self-Direction), Social (SOC; Leisure, Social), and Practical (PR; Community Use, Home Living, Health and Safety, Self-Care). A Global Adaptive Composite (GAC) score is also calculated from all nine skill area scores. Composite scores are presented as norm-referenced standard scores $(\mathrm{M}=100 ; \mathrm{SD}=15)$.

3. The Social Responsiveness Scale (SRS)—The SRS (Constantino \& Gruber, 2005) is a 65 item standardized questionnaire regarding behaviors that are associated with autism spectrum disorders. Normative data was collected on 1636 individuals in several states of the US. The temporal stability of the SRS was measured over a 17 month time period and found to be adequate with $t$ time 1-time 2 correlations between .77 and .85 . There is evidence of convergent validity with the ADI (Constantino et al., 2003). The SRS was filled out by parents to rate behaviors associated with ASD in both participant groups. We used the SRS total raw score to examine the relationship between autistic behaviors and adaptive functioning.

\section{Data Analytic Plan}

Based on the prediction that the ASD group would show lower adaptive functioning scores on the ABAS than the TD group, data were analyzed with: 1) a one-way ANOVA comparing GAC scores, 2) a 2 (group) $\times 3$ (ABAS composite scores) mixed model ANOVA), and 3) a 2 (group) $\times 9$ (ABAS skill area scores) mixed model ANOVA.

Second, ASD and TD skill area score profiles were compared using chi-square analyses. We first created individual participant score profiles by computing the differences between each participant's individual skill area scores and his/her mean skill area score (average of all nine skill area scores) following standard procedures in the ABAS-II manual. The ABAS-II manual also provides critical values (Table B.8) for each skill area at which a participant's difference score indicates a significant discrepancy $(p<.05)$ between that participant's skill area score and his or her skill area average. We used these critical values to determine which participants had skill area scores significantly higher or lower than their own average (e.g., if participant $X$ has a Social skill area score of 5 and a mean skill area score of 8 , his/her 
Social difference score is $5-8=-3$; the absolute value of this discrepancy score, 3 , is greater than the critical value of 2.22 for social skills revealing a significant individual weakness in the Social skill area). We then used chi-square analyses to compare the number of ASD and TD participants in each skill area whose difference score exceeded the critical value for that skill area, showing a significant relative weakness or strength in that skill area.

We also examined behavioral and cognitive correlates of the ABAS. We used ABAS composite scores to reduce the number of comparisons made. Pearson correlations were run between ABAS composite scores and age, IQ, and SRS for the ASD and TD groups separately. In addition, Pearson correlations between ADI and ADOS and the ABAS were calculated for the ASD group only. Because IQ correlated with some ABAS composite scores in each group, we also report partial correlations controlling for IQ.

\section{Results}

A detailed examination of the data revealed no outliers on any measure. As predicted, individuals with ASD were rated as having overall lower adaptive behavior ratings (GAC scores) than TD individuals $(F(1,68)=109.19, p<.001)$. The mixed model ANOVAs revealed main effects of group with ASD participants scoring lower on all of the ABAS composite scores $(F(1,68)=93.11, p<.001)$ and in all of the skill areas $(F(1,68)=101.71, p<.001)$ than the TD participants; however, there were no significant group by composite/skill area interactions $(F(2,67)=0.24, p=.78$ and $F(8,61)=1.61, p=.14$, respectively) (see Table 2$)$.

Figure 1 shows the skill area score profiles of the ASD and TD groups. For each skill area, the figure shows the percentage of ASD and TD individuals exhibiting a significant weakness in that area relative to the other skill areas (i.e., the participant's difference score in that skill area exceeded the critical value reported in the ABAS manual for that skill area). Chi-square analyses comparing the number of ASD and TD participants with significant weaknesses in each skill area showed that significantly more ASD than TD individuals exhibited substantial weakness in the Social skill area (Fisher's Exact Test; $p=.002$ ). There were no significant differences between the groups in any other skill area.

Age did not correlate with any of the ABAS composite standard scores in the ASD group and only with the CON composite score in the TD group (see Table 3). IQ correlated with the ABAS CON composite score in only the ASD group (medium effect size (McCarthy et al., 1991); (see Table 3). The SRS was negatively correlated with all of the ABAS composite scores in the TD group (large effect size), but only with the ABAS CON composite score in the ASD group. However, this correlation fell below the $p=.05$ significance threshold after partialling the effects of IQ (see Table 3).

Within the ASD group, a number of correlations with medium effect sizes were found between the ABAS composite scores and diagnostic ratings from the ADOS. ADOS Communication showed significant negative correlations with all four ABAS composite scores while ADOS Social was negatively correlated with the GAC and SOC composite scores from the ABAS. In contrast, ADOS Stereotyped Behaviors showed no correlations with the ABAS (see Table 4).

\section{Discussion}

In the present study we sought to characterize the adaptive behavior profile of individuals with ASD using the ABAS checklist. We found compromised levels of adaptive behavior in high-functioning individuals with ASD in all domains of adaptive behavior measured by the ABAS: conceptual, social, and practical. Based on the ABAS, individuals with ASD show relatively greater impairment in social skills, even in the context of overall impaired 
adaptive functioning. IQ was significantly associated only with the conceptual composite score in the ASD group. Higher ratings of ASD social and communication symptoms on the ADOS generally were associated with lower ABAS composite scores. Greater endorsement of ADOS communication symptoms was associated with lower ABAS composite scores across the board while increased ADOS social symptoms was associated with greater adaptive difficulties on the global and social composite scores from the ABAS. Higher autism behavior ratings as measured by the SRS, on the other hand, related negatively to ABAS adaptive behavior in the TD but not ASD group. In our sample, age did not predict ABAS scores in the ASD group and only related to the conceptual composite score in the TD group.

Overall, these findings are consistent with predictions and prior findings of adaptive deficits in ASD. Both the VABS and the ABAS document compromised levels of adaptive behavior across domains in high-functioning individuals with ASD. The particularly striking deficit in socialization found using the ABAS conforms with previous findings and affirms the ability of the ABAS to identify this pathognomonic feature of ASD (e.g., Bolte \& Poustka, 2002; Carter et al., 1998). It should be noted however that while the Social skill area was a relative weakness for the ASD group the Social composite score (SOC) was not. It is made up of the Leisure and Social skill areas. The Social skill area contains questions about friendships, pragmatic language skills, awareness of feeling states, politeness skills, and social judgment. In contrast, the Leisure skill area taps many non-social skills, such as: "Plays alone with toys, games, or other fun activities;" "Looks at pictures or reads books or magazines during free time;" "Listens to music for fun and relaxation;" and "Has a hobby or creative activity that requires making or building something;" in addition to other questions which assess a person's ability to play with others (e.g., waiting one's turn). The inclusion of these items in the SOC composite score may mean that it underestimates social adaptive deficits in ASD overall.

Some groups have found standard scores of adaptive behavior to decrease over time in ASD (Klin et al., 2007; but see Perry et al., 2009); however, we found no significant relationship between age and ABAS scores in the ASD sample. This is not surprising given the absence of children under the age of 12 in the present study. Klin and colleagues describe plateaus in adaptive behavior in later childhood and adolescence, which could be difficult to detect in a primarily adolescent and young adult sample such as ours. Similarly, we did not expect IQ to predict social adaptive behavior in the high-functioning ASD group based on the magnitude of impairment in social ability in ASD relative to IQ and based on previous findings (Freeman et al., 1999; Liss et al., 2001; Schatz \& Hamdan-Allen, 1995).

We find that higher ratings of autism communication and social symptoms relate to lower adaptive behavior functioning, which contrasts with Klin et al.'s (2007) report of weak negative relationships between symptoms and adaptive behavior, but is consistent with other reports of a strong negative association between the symptoms and adaptive behavior (Liss, et al., 2001; Perry et al., 2009). Interestingly, the SRS related strongly to adaptive functioning only in the TD group. SRS associations showed no such predictive value in the ASD group. It may be that the instrument used to measure symptomatology is highly relevant to predicting adaptive ability. Higher ADOS symptom tallies related to lower adaptive ability in our data, and Perry and colleagues (2009) found the same pattern using the Childhood Autism Rating Scale (CARS) to measure symptomatology. The SRS is a different type of measure, however, designed to capture autistic behaviors in the broad population. The SRS has not been typically used to assess the relationship between symptoms and adaptive behavior in ASD. Although there is a previous report of a strong negative correlation between SRS and VABS scores in a group of preschoolers (Pine, Luby, Abbacchi, \& Constantino, 2006), only about two-thirds of the sample had an ASD. 
The primary limitation of this study is our failure to collect VABS data along with the ABAS data for the purposes of directly comparing the two measures in the same population. We are unaware of investigations comparing the VABS and ABAS outside of data presented in their respective manuals and one investigation of adaptive behavior in epilepsy (Hamilton, Burns, \& Neale, 2005). The data reported in the both the ABAS-II and VABS-II manuals on non-clinical samples of children in similar age ranges to those reported in this study (sample sizes are 45 and 82 respectively) indicate a strong relationship between the global adaptive scores on the ABAS and the VABS (correlations $=.70$ and .78 , respectively); and related domains of the two measures (e.g., correlation of .68 between the Socialization domains/composites of the two measures). Variability among individuals is much greater in ASD than in typically developing children, however, and a direct comparison of the two measures in a group of children with ASD would be a useful future investigation. A related limitation is the lack of an interview version of the ABAS-II with which to compare the checklist form for consistency. Other limitations include lack of inclusion of lower functioning or pre-adolescent children and the small number of females in our sample, which preclude the application of our findings to those groups. The absence of other clinical groups in our study limits our knowledge of the specificity of our findings. Future investigations should include not only a second measure of adaptive functioning, but also children of all ages and intellectual levels with a range of developmental and acquired disorders.

The ASD adaptive behavior profile reveals much greater impairment than would otherwise be expected for individuals of average, and often very high, intelligence. Impaired adaptive behavior, particularly in the social domain, where IQ is not predictive, is an insidious feature of ASD that causes great struggles in daily life and interferes with independent living. Understanding the nature of these struggles is important to better understand their origin, inform ASD diagnosis, identify targets for intervention and improve outcome. The present study delineates pervasive adaptive behavior deficits among individuals with highfunctioning ASD using the ABAS, a caregiver completed checklist of adaptive functioning. It demonstrates that a relatively quickly administered questionnaire yields useful information about the adaptive skill profile in ASD and its relationship to autism symptomatology and IQ. Taken together, these results provide evidence for the effectiveness of using parent checklist reports of adaptive functioning such as the ABAS or the checklist version of the VABS-II, which can be of great utility in clinical and research settings in which a comprehensive adaptive behavior interview is not possible.

\section{Acknowledgments}

The authors were supported by the NIH, National Institute of Mental Health Intramural Research Program, during completion of this work.

The authors thank the children and their families who participated in this investigation.

\section{References}

Bassin, CH.; Gorman, PW. Overall adaptive functioning deficits in adults with Asperger's disorder: individual and mean D-KEFS, ABAS, and general neuropsychological profiles of 10 such patients. National Academy of Neuropsychology 23rd Annual Meeting; Dallas, Texas. 2003.

Black DO, Wallace GL, Sokoloff J, Kenworthy L. Brief Report: IQ split predicts social symptoms and communication abilities in high functioning children with autism spectrum disorders. Journal of Autism and Developmental Disorders. 2009; 39:1613-1619. [PubMed: 19572193]

Bolte S, Poustka F. The relation between general cognitive level and adaptive behavior domains in individuals with autism with and without co-morbid mental retardation. Child Psychiatry \& Human Development. 2002; 33:165-172. [PubMed: 12462353] 
Carter AS, Volkmar FR, Sparrow SS, Wang J, Lord C, Dawson G, Fombonne E, Loveland K, Mesibov G, Schopler E. The Vineland Adaptive Behavior Scales: Supplementary norms for individuals with autism. Journal of Autism and Developmental Disorders. 1998; 28:287-302. [PubMed: 9711485]

Constantino JN, Davis SA, Todd RD, Schindler MK, Gross MM, Brophy SL, Metzger LM, Shoushtari CS, Splinter R, Reich W. Validation of a brief quantitative measure of autistic traits: comparison of the social responsiveness scale with the autism diagnostic interview-revised. Journal of Autism and Developmental Disorders. 2003; 33:427-433. [PubMed: 12959421]

Constantino, JN.; Gruber, CP. Social Responsiveness Scale (SRS). Los Angeles, CA: Western Psychological Association; 2005.

Fenton G, D’Ardia C, Valente D, Del Vecchio I, Fabrizi A, Bernabei P. Vineland adaptive behavior profiles in children with autism and moderate to severe developmental delay. Autism. 2003; 7:269287. [PubMed: 14516060]

Freeman BJ, Del'Homme M, Guthrie D, Zhang F. Vineland Adaptive Behavior Scale scores as a function of age and initial IQ in 210 autistic children. Journal of Autism and Developmental Disorders. 1999; 29:379-384. [PubMed: 10587884]

Gillham JE, Carter AS, Volkmar FR, Sparrow SS. Toward a developmental operational definition of autism. Journal of Autism and Developmental Disorders. 2000; 30:269-278. [PubMed: 11039854]

Hamilton WG, Burns TG, Neale KM. Correlational analysis of the Vineland Adaptive Behavior Scales and the ABAS-II in children with epilepsy. Archives of Clinical Neuropsychology. 2005; 20:827827.

Harrison, PL.; Oakland, T. Adaptive Behavior Assessment System. 2. San Antonio, TX: Harcourt Assessment, Inc; 2003.

Kenworthy LE, Black DO, Wallace GL, Ahluvalia T, Wagner AE, Sirian LM. Disorganization: the forgotten executive dysfunction in high functioning autism (HFA) spectrum disorders. Developmental Neuropsychology. 2005; 28:809-827. [PubMed: 16266250]

Klin A, Saulnier CA, Sparrow SS, Cicchetti DV, Volkmar FR, Lord C. Social and communication abilities and disabilities in higher functioning individuals with autism spectrum disorders: The Vineland and the ADOS. Journal of Autism and Developmental Disorders. 2007; 37:748-759. [PubMed: 17146708]

Lainhart JE, Bigler ED, Bocian M, Coon H, Dinh E, Dawson G, Deutsch CK, Dunn M, Estes A, Tager-Flusberg H, Folstein S, Hepburn S, Hyman S, McMahon W, Minshew N, Munson J, Osann K, Ozonoff S, Rodier P, Rogers S, Sigman M, Spence MA, Stodgell CJ, Volkmar F. Head circumference and height in autism: a study by the Collaborative Program of Excellence in Autism. American Journal of Medical Genetics. 2006; 140:2257-74. [PubMed: 17022081]

Lecouteur A, Rutter M, Lord C, Rios P, Robertson S, Holdgrafer M, Mclennan J. Autism Diagnostic Interview - a standardized investigator-based instrument. Journal of Autism and Developmental Disorders. 1989; 19:363-387. [PubMed: 2793783]

Liss M, Harel B, Fein D, Allen D, Dunn M, Feinstein C, Morris R, Waterhouse L, Rapin I. Predictors and correlates of adaptive functioning in children with developmental disorders. Journal of Autism and Developmental Disorders. 2001; 31:219-230. [PubMed: 11450820]

Lord C, Risi S, Lambrecht L, Cook EH, Leventhal BL, DiLavore PC, Pickles A, Rutter M. The Autism Diagnostic Observation Schedule-Generic: a standard measure of social and communication deficits associated with the spectrum of autism. Journal of Autism and Developmental Disorders. 2000; 30:205-223. [PubMed: 11055457]

Lord C, Rutter M, Lecouteur A. Autism Diagnostic Interview-Revised - a revised version of a diagnostic interview for caregivers and individuals with possible developmental disorders. Journal of Autism and Developmental Disorders. 1994; 24:659-685. [PubMed: 7814313]

McCarthy PL, Cicchetti DV, Sznajderman SD, Forsyth BC, Baron MA, Fink HD, Czarkowski N, Bauchner H, Lustman-Findling K. Demographic, clinical and psychosocial predictors of the reliability of mothers' clinical judgments. Pediatrics. 1991; 88:1041-1046. [PubMed: 1945609]

Perry A, Flanagan HE, Dunn Geier J, Freeman NL. Brief Report: The Vineland Adaptive Behavior Scales in young children with autism spectrum disorders at different cognitive levels. Journal of Autism and Developmental Disorders. 2009; 39:1066-1078. [PubMed: 19234777] 
Pfeiffer B, Kinnealey M, Reed C, Herzberg G. Sensory modulation and affective disorders in children and adolescents with Asperger's disorder. The American Journal of Occupational Therapy. 2005; 59:335-345. [PubMed: 15969281]

Pine E, Luby J, Abbacchi A, Constantino JN. Quantitative assessment of autistic symptomatology in preschoolers. Autism. 2006; 10:344-352. [PubMed: 16908478]

Schatz J, Hamdan-Allen GJ. Effects of age and IQ on adaptive behavior domains for children with autism. Journal of Autism and Developmental Disorders. 1995; 25:51-60. [PubMed: 7608034]

Thurm A, Lord C, Lee L, Newschaffer C. Predictors of language acquisition in preschool children with autism spectrum disorders. Journal of Autism and Developmental Disorders. 2007; 37:1721-1734. [PubMed: 17180717]

Tomanik SS, Pearson DA, Loveland KA, Lane DM, Shaw JB. Improving the reliability of autism diagnoses: Examining the utility of adaptive behavior. Journal of Autism and Developmental Disorders. 2007; 37:921-928. [PubMed: 17006778]

Volkmar FR, Sparrow SS, Goudreau D, Cicchetti DV, Paul R, Cohen DJ. Social deficits in autism - an operational approach using the Vineland-Adaptive Behavior Scales. Journal of the American Academy of Child and Adolescent Psychiatry. 1987; 26:156-161. [PubMed: 3584011] 
Relative Weaknesses in ABAS Skill Areas:

Percentage of subjects below cutoff criteria

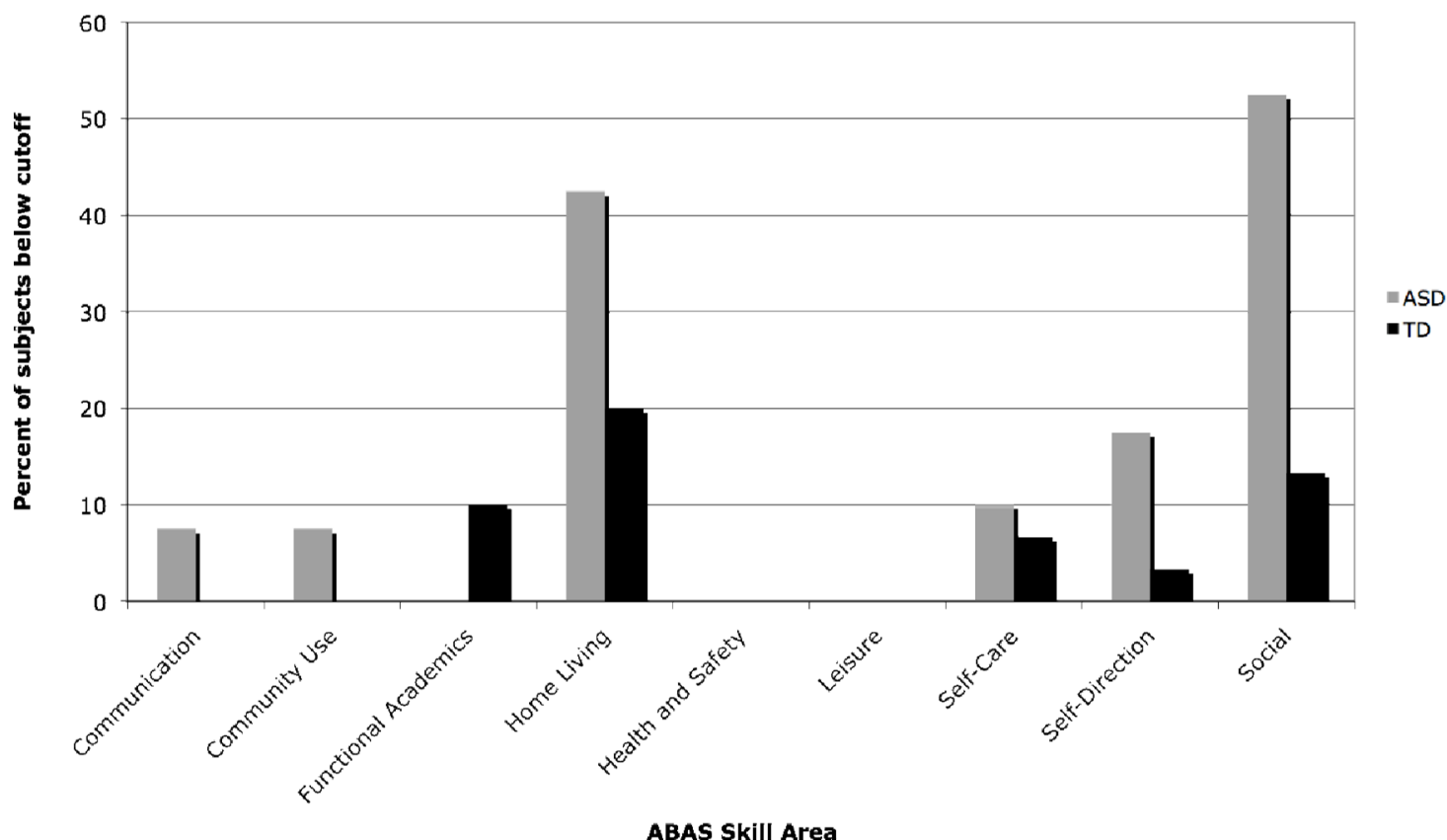

Figure 1. ABAS relative skill area weaknesses by group

Each participant's mean skill area score was subtracted from each of his/her skill area scores. The resulting individual difference scores were compared with the cut off values supplied in the ABAS manual denoting scores that deviate significantly from the participant's mean score, suggesting relative strength or weakness in a skill area. The percentage of participants in each diagnostic group with a relative weakness in a given skill area is shown here. 
Table 1

Demographic characteristics of participants.

\begin{tabular}{|l|c|c|c|c|}
\hline & $\begin{array}{c}\text { ASD [n=40] } \\
\text { Mean (SD) }\end{array}$ & $\begin{array}{c}\text { TD [n=30] } \\
\text { Mean (SD) }\end{array}$ & Statistic & $\boldsymbol{p}$ \\
\hline Age (years) & $15.33(2.46)$ & $15.99(1.92)$ & $F(1,71)=2.14$ & .15 \\
\hline Full Scale IQ & $111.75(16.24)$ & $112.83(11.15)^{*}$ & $F(1,66)=0.10$ & .76 \\
\hline Sex $(\%$ male) & $90 \%$ & $86.7 \%$ & Fisher's Exact Test & .72 \\
\hline ADI Social Interaction & $19.66(4.92)^{\bullet}$ & ----- & ----- & ---- \\
\hline ADI Verbal Communication & $15.32(4.51)^{\bullet}$ & ----- & ----- & ----- \\
\hline ADI Repetitive Behaviors & $6.00(2.93)^{\bullet}$ & ----- & ----- & ----- \\
\hline ADOS Social Interaction & $7.54(3.18)^{\psi}$ & ----- & ----- & ----- \\
\hline ADOS Communication & $3.62(1.75)^{\psi}$ & ----- & ----- & ----- \\
\hline ADOS Stereotyped Behaviors & $1.42(1.70)^{\psi}$ & ----- & ---- & ---- \\
\hline
\end{tabular}

* $\mathrm{n}=29$

$\dot{\mathrm{n}}=38$

$\psi_{\mathrm{n}}=37$ 
Table 2

ABAS composite and skill area scores by group.

\begin{tabular}{|c|c|c|}
\hline & $\operatorname{ASD}[n=40]$ & TD $[n=30]$ \\
\hline & Mean (SD) & Mean (SD) \\
\hline \multicolumn{3}{|c|}{ Composite Scores (std scores; $100 \pm 15$ ) } \\
\hline Global Adaptive Composite & $72.10(13.33)$ & $106.47(14.00)$ \\
\hline Conceptual & $79.57(12.74)$ & $107.27(12.54)$ \\
\hline Social & $73.47(11.01)$ & $102.60(13.99)$ \\
\hline Practical & $73.05(16.49)$ & $101.90(13.16)$ \\
\hline \multicolumn{3}{|l|}{ Skill Areas (scaled scores; $10 \pm 3$ ) } \\
\hline Communication & $5.92(2.12)$ & $11.37(1.90)$ \\
\hline Community Use & $6.32(3.24)$ & $11.10(2.09)$ \\
\hline Functional Academics & $7.07(3.05)$ & $11.30(2.90)$ \\
\hline Home Living & $4.05(3.25)$ & $9.40(3.50)$ \\
\hline Health and Safety & $6.55(3.33)$ & $11.17(1.80)$ \\
\hline Leisure & $6.27(2.39)$ & $11.10(2.62)$ \\
\hline Self-Care & $5.22(3.12)$ & $10.10(2.09)$ \\
\hline Self-Direction & $5.65(3.10)$ & $10.90(2.47)$ \\
\hline Social & $3.40(2.72)$ & $9.80(2.75)$ \\
\hline
\end{tabular}




\section{Table 4}

Pearson correlations between ABAS composite scores and ADOS scores within the group of ASD individuals (partialling out the effects of IQ).

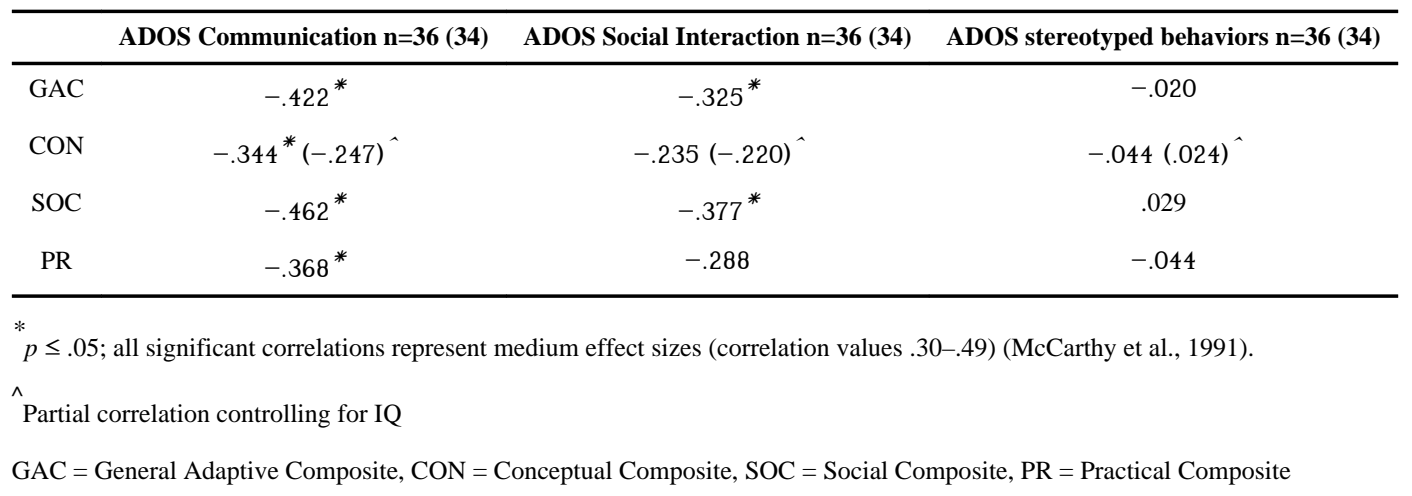

